\title{
Importance of QC for the reliable preparation of NGS libraries
}

\author{
Advanced Analytical Technologies, Inc. 2450 SE Oak Tree Ct. Ankeny, IA 50021 USA www.aati-us.com
}

\section{Introduction}

Nucleic acid QC is an essential component of next-generation sequencing workflows. Confirming nucleic acid quality at checkpoints throughout NGS library preparation - from incoming nucleic acid QC to final library validation - helps ensure successful sequencing outcomes.

Advanced Analytical Technologies, Inc. (AATI) addresses the QC needs of researchers performing NGS with a combination of parallel capillary electrophoresis and fluorescence detection of nucleic acids. The Fragment Analyzer ${ }^{\text {Tw }}$ Automated CE System uses thoughtful design considerations to provide superior nucleic acid detection and separation capacity, while maintaining ease-of-use. Easily size, quantify, and qualify DNA and RNA samples without swapping capillary arrays, and complete analytic separations in about one hour with the gold standard of nucleic acid QC.

\section{Incoming Nucleic Acid QC}

NGS commonly begins with isolation of genomic DNA or total RNA. Knowing the quality of incoming nucleic acids prior to NGS library construction is crucial. Understanding the quality of DNA and RNA samples helps ensure optimal NGS library preparation, in turn supporting successful sequencing. Reliable QC of DNA and RNA samples requires an accurate understanding of the size and concentration, as well as visual confirmation of sample quality.

\section{Genomic DNA}

Genomic DNA is a common starting point for numerous NGS applications. QC analysis of gDNA is frequently complicated by the high molecular weight of the sample, impeding sizing and quantification. By employing a combination of specially designed separation matrices and carefully programmed separation methods, the Fragment Analyzer line of instruments can reliably separate gDNA (Figure 1) with accurate sizing through $60 \mathrm{~Kb}$ in about one hour.

Objective QC analysis of gDNA samples post-electrophoresis is aided by the Genomic Quality Number (GQN), a dynamic quality metric available in $P R O S i z e^{\circledR}$ Data Analysis Software. Calculated from a user-defined Size Threshold, the GQN reflects the quantity of gDNA that meets unique, user-defined standards. Reported on a scale of 0 to 10 , a higher value indicates that more of the gDNA sample falls above the Size Threshold.

FFPE gDNA is a valuable resource, though it presents unique QC challenges. The GQN can quickly evaluate the suitability of FFPE gDNA samples separated on the Fragment Analyzer. Degraded by tissue preservation, FFPE gDNA samples evade accurate analysis. The Fragment Analyzer provides complete separation of these samples, while the GQN allows researchers to define what constitutes suitable size for FFPE samples moving on to NGS'.

\section{Total RNA}

Total RNA (Figure 2) contains a wealth of information ready for mining with NGS strategies. QC analysis of total RNA is crucial, as use of degraded or otherwise low-quality samples for NGS library construction can compromise success. Challenges with total RNA QC

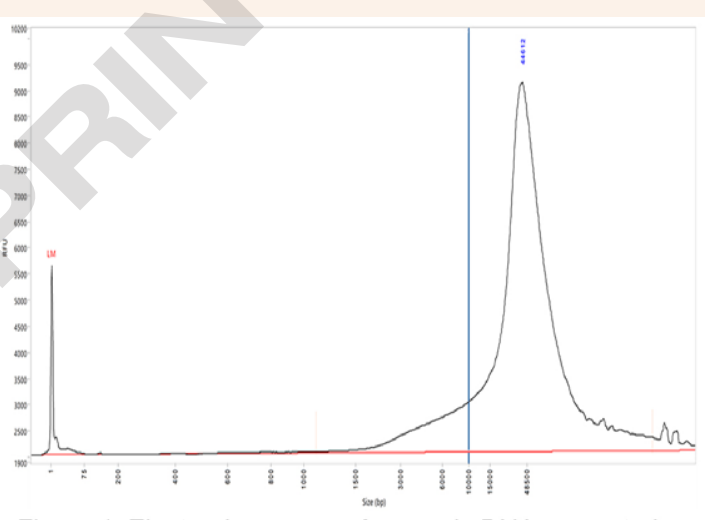

Figure 1. Electropherogram of genomic DNA separated on the Fragment Analyzer ${ }^{\mathrm{TM}}$ Automated CE System. $\mathrm{GQN}=8.6$ with a Size Threshold of 10,000 bp (blue line).

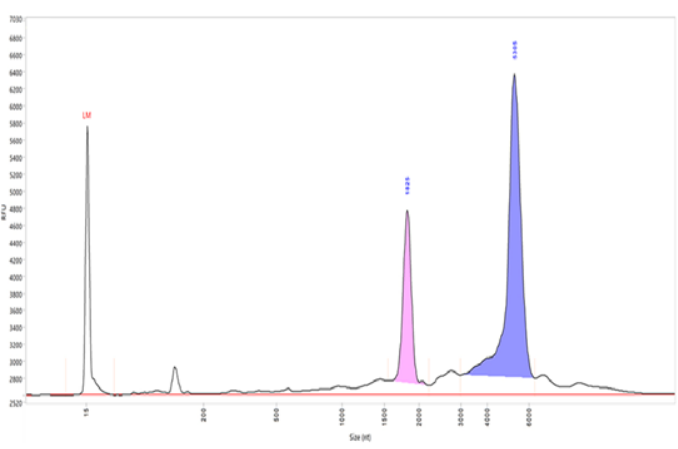

Figure 2. Electropherogram of total RNA separated on the Fragment Analyzer ${ }^{\mathrm{TM}}$ Automated CE System. RQN $=9.9$ 


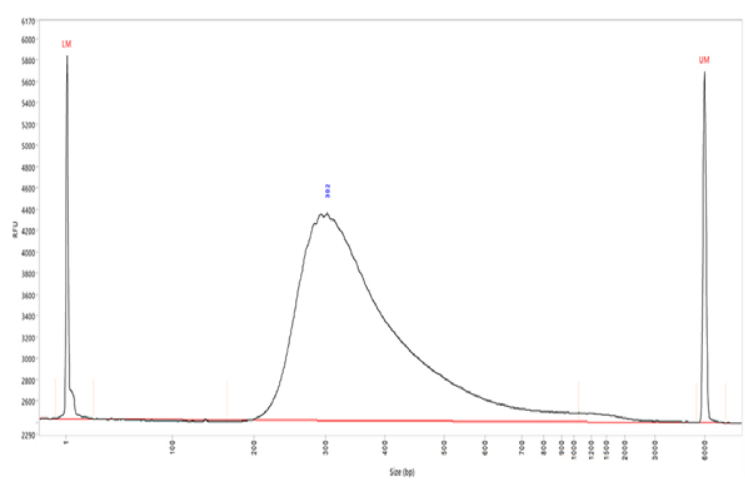

Figure 3. Electropherogram of a short-read library separated on the Fragment Analyzer ${ }^{\mathrm{TM}}$ Automated CE System.

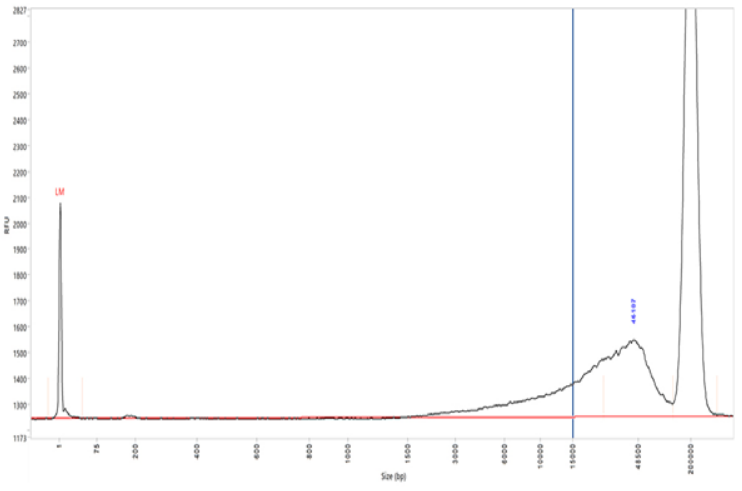

Figure 4. Electropherogram of a large-insert library separated on the Fragment Analyzer ${ }^{\mathrm{TM}}$ Automated CE System. GQN $=7.1$ with a Size Threshold of $15,000 \mathrm{bp}$

stem from poor separation quality, limiting accurate sizing and quantification of important regions, including the Fast Region, small rRNA subunits, and large rRNA subunits. Superior separation parameters and exceptional sensitivity delivered by the Fragment Analyzer provide researchers with a complete picture of total RNA quality, helping ensure only suitable samples proceed to downstream analysis.

Post-separation analysis of total RNA is greatly simplified by PROSize. Using the RNA Quality Number (RQN), researchers are able to quickly, and objectively, determine which total RNA samples are suitable for NGS library preparation. Automatically provided by PROSize in the RNA Property Summary, the RQN is reported on a scale of one to 10, with higher values corresponding to higher quality total RNA.

While the RQN delivers accurate objective analysis of total RNA expected to be of good quality, it has limited application with FFPE RNA samples often studied in clinical research settings. To this end, the $\mathrm{DV}_{200}$ quality metric was developed ${ }^{2}$. Available for PROSize, the $\mathrm{DV}_{200}$ reports the percentage of RNA fragments that exceed $200 \mathrm{nt}$ in size. Notably, this quality metric has been found to have a direct correlation with a samples relative performance in NGS workflows. The $\mathrm{DV}_{200}$ helps researchers obtain critical results from FFPE RNA sequencing projects by ensuring only suitable samples proceed to NGS.

Final Library Validation

Final QC analysis of NGS libraries prior to sequencing ensures that library construction was successful. It is also when researchers will calculate the molarity of the library from its measured average size and concentration. NGS flow cells require precision loading to help guarantee the generation of high-quality sequencing results. The Fragment Analyzer can simultaneously provide visual confirmation of library quality, and calculate molarity for short-read and long-read NGS libraries.

Short-read NGS (Figure 3) is perhaps one of the most common sequencing strategies in use today. While chemistries differ, all short-read NGS relies on the use of libraries comprised of relatively short DNA fragments. Frequently employed in high-throughput workflows, shortread NGS demands a QC solution that can adapt to different throughput demands without sacrificing accuracy or precision. The Fragment Analyzer automates QC analysis, processing 12, 48, or 96 samples in an hour and up to 288 samples without researcher intervention.

In contrast to short-read NGS, long-read NGS is characterized by employing large-insert libraries. These libraries can use DNA inserts tens of kilobases long (Figure 4), simplifying post-sequencing assembly and analysis. While long-read sequencing has its benefits, it also has QC challenges. Primarily, the size of the library defies accurate sizing and quantification by legacy methods. The Fragment Analyzer can size and quantify DNA smears, including long-read NGS libraries up to $48.5 \mathrm{~Kb}$, in about 1 hour, considerably faster and with higher accuracy and precision than competing instruments.

\section{Summary}

The Fragment Analyzer is the gold standard of nucleic acid QC for NGS workflows, simplifying necessary QC analysis from incoming nucleic acids to final library validation. DNA and RNA samples are easily separated using the same capillary array, while automated instrument operation and parallel capillary electrophoresis bring researchers an efficient, accurate, and easy-to-use QC solution.

\section{References}

\section{${ }^{1}$ Evaluating DNA Quality from FFPE Samples}

https://www.illumina.com/content/dam/illumina-marketing/documents/products/technotes/truseq-exome-ffpe-technote-770-2015-035.pdf

${ }^{2}$ Evaluating RNA Quality from FFPE Samples, Illumina ${ }^{\circledR}$.

https://www.illumina.com/content/dam/illumina-marketing/documents/products/technotes/evaluating-rna-quality-from-ffpesamples-technical-note-470-2014-001.pdf 